\title{
Identification of multiple dmrt1s in catfish: localization, dimorphic expression pattern, changes during testicular cycle and after methyltestosterone treatment
}

\author{
K Raghuveer and B Senthilkumaran \\ Department of Animal Sciences, School of Life Sciences, University of Hyderabad, PO Central University, Gachibowli, Hyderabad 500 046 , Andhra Pradesh, India \\ (Correspondence should be addressed to B Senthilkumaran; Email: bsksI@uohyd.ernet.in)
}

\begin{abstract}
The double sex and mab-3 related (DM) transcription factor 1 (dmrt1) plays an important role in testicular differentiation. Here, we report cloning of multiple dmrt1s, a full-length and two alternative spliced forms from adult catfish (Clarias gariepinus) testis, which encode predicted proteins of 287 (dmrt1a), 253 (dmrt1b) and 233 (dmrt1c) amino acid residues respectively. Interestingly, $d m r t 1 c$ lacks the majority of the DM domain. Multiple dmrt1s (dmrt1a and dmrt1c) were obtained from Clarias batrachus as well. Tissue distribution (transcript and protein) of catfish dmrt1 revealed exclusive expression in testis. Semi-quantitative RT-PCR revealed the presence of multiple dmrt1s with high levels of $d m r t 1 a$ in adult testis but not in ovary. Real-time RT-PCR analysis during testicular cycle showed higher levels of $d m r t 1$ transcripts in preparatory and pre-spawning when compared with spawning and post-spawning phases. Immunocytochemical and immunofluorescence localization revealed the presence of catfish Dmrt1 protein in spermatogonia and spermatocytes, which indicates plausible role in spermatogenesis. Histological analysis indicated initiation of gonadal sex differentiation in catfish around $40-50$ days after hatching. The potential role for dmrt1 in testicular differentiation is evident from its stage-dependent elevated expression in developing testis. Furthermore, dimorphic expressions of $d m r t 1 s$ were evident at different stages of gonadal development or recrudescence in catfish. Treatment of methyl testosterone (MT) during early stages of gonadal sex differentiation resulted in adult males. Interestingly, we also obtained MT-treated fishes having ova-testis gonads. Analysis of dmrt1, sox9a, foxl2 and cyp19a1 expression patterns in MT-treated gonads revealed tissue-specific pattern. These results together suggest that multiple dmrt1s are testis-specific markers in catfish.
\end{abstract}

Journal of Molecular Endocrinology (2009) 42, 437-448

\section{Introduction}

Several transcription factors like sry, $d m y$, sox9, $a d 4 B P / S F-1, d m r t 1, d a x 1$ and $w t 1$ have been identified to play an important role in sex determination and differentiation of vertebrates including teleosts (Swain \& Lovell-Badge 1999, Hughes 2001, Matsuda et al. 2002, Wang et al. 2002, Kobayashi et al. 2004). Molecular similarity in sexual development across different phyla found so far is among Drosophila doublesex, Caenorhabditis $m a b-3$ and vertebrate $d m r t 1$ (Raymond et al. 1998). Dsx and $m a b-3$ related transcription factor 1 ( $d m r t 1)$ belongs to gene family of putative transcription factors that share a highly conserved novel zinc finger DNA binding domain (DM domain) across different phyla (Raymond et al. 1998). Dmrt1 has been cloned from several vertebrate species including mammals, birds, reptiles, amphibians and fishes (Nanda et al. 1999, De Grandi et al. 2000, Guan et al. 2000, Kettlewell et al. 2000, Nagahama 2005, Osawa et al. 2005) and has been implicated in testicular differentiation. Furthermore, dmrt1 has been localized in 9p23.4 and monosomy at this region in $\mathrm{XY}$ individuals and manifests feminization and gonadal dysgenesis (Ottolenghi \& McElreavey 2000). In mice, Dmrt1 expresses in both male and female embryonic genital ridges, but as the differentiation proceeds, it is gradually lost from the ovary and expressed only in the Sertoli and germ cells of testis. High expression of Dmrt1 was observed in testis at 13.5 days post coitum and its expression is maintained throughout the adult after birth (Raymond et al. 1999, De Grandi et al. 2000). Localization of Dmrt1 in the Z-chromosome of birds designated it as a male master testis-determining gene (Nanda et al. 1999). Dmrt1 knockout mice showed its dispensability in females yet it is required in males for postnatal testicular development by effecting the differentiation of both Sertoli and germ cells (Raymond et al. 2000). These expression patterns indicate that $d m r t 1$ is likely to have a conserved role in the early stages of testis development. Studies from lower vertebrates are mostly done in teleosts to specify the conserved role of $d m r t 1$. $D M Y$, a Y-linked male sex-determination gene, similar to sry discovered in medaka has been shown to be a duplicate of autosomal gene dmrt1 (Matsuda et al. 2002, Zhang 2004). Nevertheless, most of these reports are

DOI: 10.1677/JME-09-0011 Online version via http://www.endocrinology-journals.org 
from daily or fortnight breeders like zebrafish, medaka and tilapia (Guan et al. 2000, Kobayashi et al. 2004, Guo et al. 2005). Research reports on this line using annual breeders with a focus on seasonal reproductive cycle are limited (Marchand et al. 2000, Huang et al. 2005). In this regard, fish that undergo seasonal pattern of gonadal attenuation and recrudescence rather than continuously mature individuals may provide interesting highlights to understand the role of $d m r t 1$ not only during development but also during recrudescence. The fate of $d m r t 1$ transcripts after testicular differentiation is not clear at present. Such an attempt may provide more insights to understand its role in adult, if any. Although tracking of dmrt1 may not provide a direct role to this issue but may contribute to understanding whether timing of dmrt1 expression coincides with the beginning of testicular recrudescence vis-à-vis spermatogenesis. Catfish, Clarias gariepinus is an annual breeder, which takes 1 year to attain maturity. It is domesticated in south India and interestingly it exhibits seasonal pattern of reproductive cycle. Previously, we reported cloning of partial cDNA fragments of $d m r t 1$ from catfish testis that indicated the presence of more than one form of dmrt1 (Raghuveer et al. 2005). In the present study, we aimed to clone full-length dmrt1 cDNA from catfish testis and also explored the possibility of multiple forms. In addition, we also aimed to study the spatio-temporal expression pattern of $d m r t 1(\mathrm{~s})$ during early stages of gonadal development in juveniles as well as at different phases of testicular recrudescence in adult catfish. We also intend to confirm the presence of dmrt1(s) from a closely related catfish species Clarias batrachus to augment our findings. Furthermore, our study was extended to localize dmrt1 in juvenile and adult testis. Administration of methytestosterone (MT) or ethynylestradiol (EE2) is a useful strategy to skew the sex of the population in question to study testicular and ovarian differentiation (Nagahama 2005). We used MT treatment strategy to explicitly define the role of $d m r t 1$ and endorse its importance as a candidate marker for testicular development.

\section{Materials and methods}

\section{Animals and sampling}

Catfish (C. gariepinus) at different age groups were reared in fresh water tanks under ambient photothermal conditions. Mature spermiating male and gravid female fishes were used for IVF to obtain catfish fries at different age groups. The newly hatched catfish fries were fed with live tube worms until 3 months. Juvenile fishes of 3-8 months old were fed with live tube worms and commercially available fish feed. Adult catfish (1-year old) were reared in the outdoor tanks of the laboratory and fed with minced goat liver in addition to fish feed. Commonly referred to as the African or air-breathing catfish, this species is abundantly available in the ponds and lakes of Hyderabad. The seasonal reproductive cycle of catfish is divided into four phases (Swapna et al. 2006): preparatory (February-March), prespawning (April-June), spawning (July-October) and post-spawning/regressed phases (November-January). Adult gonads during the reproductive cycle of catfish were collected and a part of it was fixed in Bouin's fixative while remaining tissue was stored at $-80^{\circ} \mathrm{C}$ for total RNA extraction. Juvenile catfish fries at different age groups (50, 100 and 150 days after hatching (dah)) were dissected and gonads were removed using fine forceps under stereozoom microscope (Leica, Wetzlar, Germany). Number of gonads of similar age group and same sex were pooled and stored at $-80^{\circ} \mathrm{C}$ for total RNA extraction. Treatments of MT were given to catfish fingerlings during the critical period of gonadal differentiation as explained earlier (Raghuveer et al. 2005). In brief, about 250 catfish fries were treated with MT in pulse $(3 \mathrm{~h})$ at a dose of $500 \mathrm{ug} / 1$ intermittently (six durations) till 21 dah. We used high doses of MT as low doses were found to be ineffective in our pilot studies. The MT-treated fishes were reared until they reach maturity and after ( 1 to 2 years). Then their gonads were dissected out for histology and total RNA extraction. Adult C. batrachus were obtained from local fish markets of Hyderabad as well as from north India. These were maintained and fed with minced goat liver ad libitum during acclimation in the laboratory for a fortnight before killing.

\section{Cloning of full-length and alternatively spliced forms of dmrt1}

Primers for rapid amplification of cDNA ends (RACE) were designed based on the sequence information of partial cDNA fragment of catfish dmrt1 that was cloned earlier. SMART $5^{\prime}$ and $3^{\prime}$ cDNA templates were made from testis total RNA according to manufacturer's protocol using the SMART RACE cDNA amplification kit (Clontech, Tokyo, Japan). 5' RACE was performed using the DM domain primer, DMr1: 5'-GCTATCTCCACTGGGCATCTGCTGGT- ${ }^{\prime}$ and universal primer A mix (UPM): 5'-CTAATACGACTCACTATAGGGCAAGCAGTGGTATCAACGCAGAGT-3'. Touchdown PCR cycling conditions were as follows: each at $94^{\circ} \mathrm{C}(30 \mathrm{~s})$ and $72{ }^{\circ} \mathrm{C}(2 \mathrm{~min})$ for 5 cycles, $94{ }^{\circ} \mathrm{C}(30 \mathrm{~s})$ and $70{ }^{\circ} \mathrm{C}$ $(2 \mathrm{~min})$ for 5 cycles, $94^{\circ} \mathrm{C}(30 \mathrm{~s}), 68^{\circ} \mathrm{C}(30 \mathrm{~s})$ and $72^{\circ} \mathrm{C}$ $(2 \mathrm{~min}$ ) for $27 \mathrm{cycles}$, in a $20 \mu \mathrm{l}$ reaction mix containing $1 \times$ PCR buffer, $100 \mu$ MdNTP mix, 1U PCR advantage Taq DNA polymerase (Clontech), $0 \cdot 1 \mu \mathrm{M}$ each primer, and $1 \mu \mathrm{l}$ of the cDNA. After the primary PCR, nested PCR was performed using $5^{\prime}$ nested primer DMr2: 
5'-AATCATTTCCTGGCTCATCCTTCACC-3' ${ }^{\prime}$ and nested universal primer (NUP): 5'-AAGCAGTGGTATCAACGCAGAGT- ${ }^{\prime}$. The nested PCR conditions were as follows: $94{ }^{\circ} \mathrm{C}(30 \mathrm{~s}), 65{ }^{\circ} \mathrm{C}(30 \mathrm{~s})$ and $72{ }^{\circ} \mathrm{C}(2 \mathrm{~min})$ for 35 cycles. $3^{\prime}$ RACE was performed using the DM domain primer DMf1: 5'-ATGCCGAAGTGCTCCC GGTGCAGG-3' , and UPM using the same PCR conditions mentioned above. After $3^{\prime}$ RACE, nested PCR was performed using $3^{\prime}$ nested primer DMf2: 5'-GTCCCGCCAGTTACAGAAGCGCTTG-3' and NUP using the same PCR conditions described above. All PCR amplifications were done using thermal cycler (Applied Biosystems, Foster City, CA, USA). All the amplified cDNA fragments were gel-purified (Qiagen, Hamburg, Germany), cloned in TOPO cloning vector (Invitrogen, Carlsbad, CA, USA) and nucleotide sequenced. We aimed to amplify the open reading frame (ORF) of dmrt1 using primers dmrt1orfF and dmrt1orfR (Table 1) designed at ORF flanking region of $d m r t 1$ cDNA obtained by RACE. The PCR conditions used for ORF amplification were as follows: $94{ }^{\circ} \mathrm{C}(30 \mathrm{~s}), 60{ }^{\circ} \mathrm{C}(30 \mathrm{~s})$ and $72{ }^{\circ} \mathrm{C}(1 \mathrm{~min})$ for 35 cycles. The PCR amplified products were gel-purified, cloned in TOPO cloning vector (Invitrogen) and nucleotide sequenced.

\section{Tissue distribution pattern of dmrt1 by reverse transcription (RT)-PCR}

RT-PCR was carried out to study expression pattern of dmrt1 in different tissues of adult catfish. For this, total RNA was extracted from different tissues (brain, spleen, gill, heart, intestine, kidney, liver, testis and ovary) of adult catfish using the Sigma TRI-reagent (Sigma)

Table 1 List of primers used for PCR amplification

Nucleotide sequence $\left(5^{\prime}-3^{\prime}\right)$

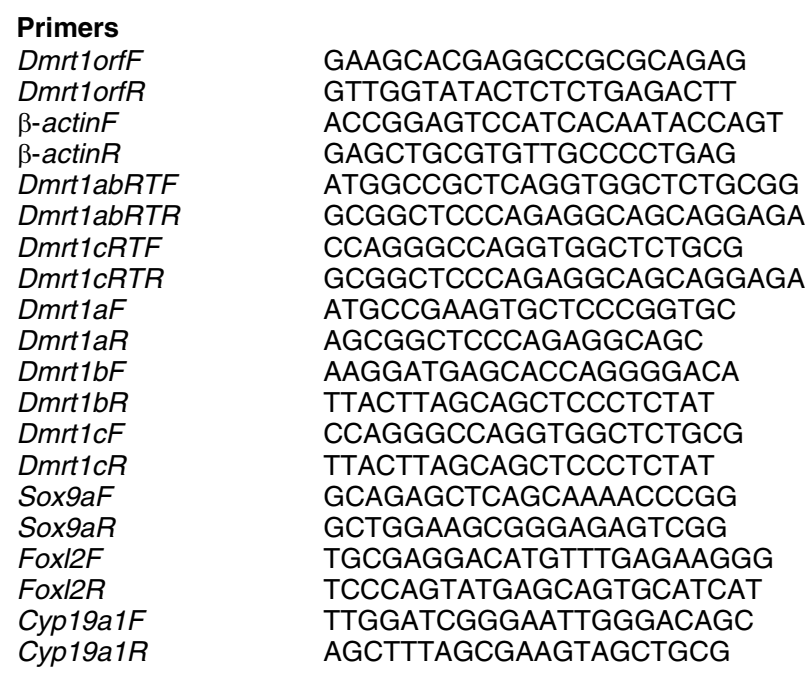

method. Reverse transcription was carried out using superscript-III reverse transcriptase (Invitrogen) with oligo $\mathrm{d}(\mathrm{T})_{18}$ primers and $5 \mu \mathrm{g}$ of total RNA at $50{ }^{\circ} \mathrm{C}$. PCR amplification was done using thermal cycler (Applied Biosystems) under the following conditions: $94^{\circ} \mathrm{C}(1 \mathrm{~min}), 58^{\circ} \mathrm{C}(30 \mathrm{~s}), 72^{\circ} \mathrm{C}(1 \mathrm{~min})$, for 35 cycles using specific $d m r t 1 a F$ and $d m r t 1 a R$ primers (Table 1 ). $\beta$-actin was used as an internal endogenous control to test the quality of cDNA templates.

\section{Expression pattern of multiple dmrt1s in developing and adult gonads by semi-quantitative RT-PCR}

Semi-quantitative RT-PCR as described by Kwon et al. (2001) was carried out to study the expression patterns of multiple $d m r t 1 s$ in adult and developing gonads. Total RNA was prepared from adult and developing gonads collected from juvenile catfish at 50, 100 and 150 dah using the Sigma TRI-reagent method. Reverse transcription was carried out using superscript-III reverse transcriptase (Invitrogen) with oligo $\mathrm{d}(\mathrm{T})_{18}$ primers and $5 \mu \mathrm{g}$ of total RNA at $50^{\circ} \mathrm{C}$. PCR amplification was done using thermal cycler (Applied Biosystems) under the following conditions: $94{ }^{\circ} \mathrm{C}$ $(1 \mathrm{~min}), 60{ }^{\circ} \mathrm{C}(30 \mathrm{~s}), 72^{\circ} \mathrm{C}(1 \mathrm{~min})$, for 30 cycles using specific primers dmrt1aF, dmrt1aR, dmrt1bF, $d m r t 1 b R, d m r t 1 c F$ and $d m r t 1 c R$ designed for amplification of respective dmrt1 forms (Table 1). Catfish $\beta$-actin was PCR amplified at $94{ }^{\circ} \mathrm{C}(1 \mathrm{~min}), 60{ }^{\circ} \mathrm{C}(30 \mathrm{~s})$, $72{ }^{\circ} \mathrm{C}(1 \mathrm{~min})$ for 28 cycles using specific $\beta$-actinF and $\beta$-actinR primers (Table 1 ) as an internal control.

\section{Real-time quantitative RT-PCR (qRT-PCR) for dmrt1s}

Pilot experiments for multiple dmrt1s expression during testicular cycle was done by semi-quantitative RT-PCR using ORF-specific primers. Later, expression of multiple $d m r t 1 \mathrm{~s}$ was analyzed by relative qRT-PCR using SYBRGreen detection method. Total RNA was extracted from the testis samples of four different phases (preparatory, pre-spawning, spawning and postspawning phases) using the Sigma TRI-reagent method, and RT was carried out using random hexamers in the presence of superscript-III reverse transcriptase (Invitrogen) according to manufacturer's protocol. Successful RT was confirmed for all samples by performing PCR amplification of internal control $\beta$-actin. Dmrt1 and $\beta$-actin specific primers were designed such that one primer spanned the junction of two exons, giving a single cDNA PCR product and precluding amplification of genomic DNA. Similarly, catfish specific $\beta$-actin $F$ and $\beta$-actinR primers (Table 1) were designed for use as normalization controls. One set of specific primers dmrt1abRTF and dmrt1abRTR (Table 1) were used to amplify the DM domain region, which will give both $d m r t l \mathrm{a}$ and $\mathrm{b}$ transcripts of same 
size except $d m r t 1 \mathrm{c}$ form. Another set of specific primers dmrt1cRTF and dmrt1cRTR (Table 1) were used to amplify exclusively $d m r t 1 c$ (DM-less form) only. Specific cDNA amplification was then carried out in triplicate using power SYBRGreen PCR Mastermix (Applied Biosystems) in a 7500 Fast Real-time PCR machine (Applied Biosystems) at $60{ }^{\circ} \mathrm{C}$ annealing temperature for 40 cycles according to the manufacturer's protocol. Melting-curve analysis was performed for each sample to check single amplification. During PCR, fluorescence accumulation resulting from DNA amplification was recorded using the sequence detector software (Applied Biosystems). Cycle threshold (CT) values were obtained from the exponential phase of PCR amplification, and $d m r t 1$ expression was normalizedagainst $\beta$-actin expression, generating a $\Delta \mathrm{CT}$ value $(\Delta \mathrm{CT}=$ $d m r t 1$ CT - $\beta$-actin CT). Relative expression was then expressed according to the Default $2^{-\Delta \mathrm{CT}}$.

\section{Production of polyclonal antibody against Dmrt1 protein}

Polyclonal anti-catfish Dmrtl antibody was raised in rabbit using catfish partial Dmrt1 recombinant protein of the conserved DM-domain as antigen. The partial Dmrt1 protein was expressed in Escherichia coli DE3 LacI host using $0.5 \mathrm{M}$ IPTG by cloning the partial dmrt1 cDNA fragment of $340 \mathrm{bp}$ into bacterial pET BLUE2 vector system (Novagen, Madison, WI, USA). The expressed fusion protein with the histidine tag (Histag) was then purified using Ni-NTA Agarose column (Qiagen) according to the manufacturer's protocol. This purified recombinant protein was used to raise polyclonal antibody in rabbit after confirming by immunoblot using the monoclonal His-tag antibody. For raising polyclonal antibody against the purified protein, rabbit was first injected with Freund's complete adjuvant (Bangalore Genei Pvt Ltd, Bangalura, India) at the vola. Later after 1 week, $500 \mu \mathrm{g}$ of the purified protein emulsified in Freund's complete adjuvant was injected into the swollen lymphoid node. Then two booster injections were given with the same antigen amount but using Freund's incomplete adjuvant (Bangalore Genei Pvt Ltd) for each week. After the last booster dose the rabbit was bled 2 weeks later and the serum sample was stored at $-80^{\circ} \mathrm{C}$ until use. Rabbit preimmune serum was obtained by collecting $2 \mathrm{ml}$ blood before antigen injection. All the rabbits in the present study were used following the guidelines of Institutional Animal Ethics Committee and Committee for the Purpose of Control and Supervision of Experiments on Animals and also after obtaining prior permission.

Western blot analysis was carried out to verify the specificity of the polyclonal Dmrt1 antibody raised in the rabbit. For this, different tissues (testis, ovary, gut, spleen, liver, muscle, heart, brain) of catfish were homogenized in $250 \mu \mathrm{l}$ homogenization buffer containing $50 \mathrm{mM}$ Tris- $\mathrm{HCl}(\mathrm{pH} 7 \cdot 4), 150 \mathrm{mM} \mathrm{NaCl}$, $1 \mathrm{mM}$ DTT, $0 \cdot 1 \%$ TritonX-100 and $0 \cdot 1 \mathrm{mM}$ phenylmethylsulphonyl fluoride using a Sigma hand homogenizer. Of each sample $50 \mu \mathrm{g}$ with a pre-stained marker was electrophoresed through a 15\% SDSpolyacrylamide gel and transferred onto nitrocellulose membrane (Pall-Life Sciences, NY, USA). Membrane was blocked in 5\% skimmed milkpowder in Trisbuffered saline with $0 \cdot 1 \%$ Tween 20 (TBST) for $1 \mathrm{~h}$ at room temperature. After blocking, membrane was washed three times for $15 \mathrm{~min}$ each in TBST solution and incubated with purified anti-catfish Dmrt1 antibody at $4{ }^{\circ} \mathrm{C}$ overnight in $0.5 \%$ skimmed milk powder/TBST solution. Blot was washed and incubated with secondary antibody alkaline phosphatase conjugated goat anti-rabbit IgG (Bangalore Genei Pvt Ltd) for $1 \mathrm{~h}$. After washing, blot was developed using BCIPNBT (Bangalore Genei Pvt Ltd).

\section{Immunocytochemical and immunofluorescence localization of $d m r t 1$}

Testis sections from adult male catfish were deparafinnised in xylene, rehydrated in successively lower graded concentrations of ethanol and then treated with $0.1 \%$ $\mathrm{H}_{2} \mathrm{O}_{2}$ for 10 mins to prevent endogenous peroxidase reaction. The sections were washed in $0 \cdot 1 \mathrm{M}$ PBS with $0 \cdot 1 \%$ Tween 20 (PBST) and then blocked with $10 \%$ normal goat serum in $0.1 \mathrm{M}$ PBS for $10 \mathrm{~min}$ at room temperature. Sections were then incubated overnight at $4^{\circ} \mathrm{C}$ in 1:1000 dilution of anti-catfish $d m r t 1$ antiserum or antiserum pre-absorbed with excess of peptide used for immunization. Later sections were washed thrice with PBST and incubated with biotin conjugated secondary antibody at room temperature for $1 \mathrm{~h}$. The sections were incubated with 1:500 dilution of streptavidin labelled HRP conjugate for $15 \mathrm{~min}$ at room temperature. The sections were washed with PBS and colour was developed using commercially supplied $3^{\prime} 3^{\prime}$-diaminobenzidine as chromogen and $\mathrm{H}_{2} \mathrm{O}_{2}$ as substrate for HRP. The sections were washed, dehydrated in graded ethanol, cleared in xylene and mounted using distrene-plasticizer-xylene mountant. Photomicrographs were taken using Olympus light microscope (Olympus, Japan). For immunofluorescence studies, testis sections were processed in the same way as mentioned above up to primary antibody incubation step except for $0.1 \% \mathrm{H}_{2} \mathrm{O}_{2}$ treatment. The sections were then in PBST and incubated with FITC fluorescent-labelled secondary antibody at room temperature for $1 \mathrm{~h}$. After that the sections were washed and mounted using $90 \%$ glycerol. Photomicrographs were taken immediately using Olympus fluorescence microscope (Olympus). Hematoxylin and propidium iodide (PI) was used as a counter stain for better clarity. 
All reagents and secondary antibodies to perform immunocytochemistry were obtained from Bangalore Genei Pvt Ltd.

\section{Expression patterns of dmrt1, sox9a, fox/2 and cyp19a1 in gonads of adult MT-treated fishes}

Total RNA was extracted from gonadal tissues of adult MT-treated catfish using the Sigma TRI-reagent method. Reverse transcription was carried out using superscript-III reverse transcriptase (Invitrogen) with oligo $\mathrm{d}(\mathrm{T})_{18}$ primers and $5 \mu \mathrm{g}$ of total RNA at $50{ }^{\circ} \mathrm{C}$. PCR amplification was done using thermal cycler (Applied Biosystems) under the following conditions: $94{ }^{\circ} \mathrm{C}(1 \mathrm{~min}), 55{ }^{\circ} \mathrm{C}(30 \mathrm{~s}), 72{ }^{\circ} \mathrm{C}(1 \mathrm{~min})$, for 35 cycles using specific primers (Table 1) designed for dmrtla, sox9a, foxl2 (Sridevi \& Senthilkumaran, unpublished observations) and cyp19a1 (Rasheeda et al. 2005) genes of $C$. gariepinus. $\beta$-actin was used as an internal control to test the quality of cDNA templates.

\section{Histology}

The trunk region of catfish fries at different stages (30, $50,75,100,150 \mathrm{dah})$ and gonads of adult MT-treated fishes were fixed in Bouin's solution, dehydrated and embedded in paraplast (Sigma). For light microscopy, $4 \mathrm{~mm}$ thick sections were cut and stained with hematxylin-eosin. All the photomicrographs for histological analysis were taken using Olympus light microscope.

\section{Results}

\section{cDNA cloning of multiple alternative transcripts of catfish dmrt1}

To isolate cDNA of dmrt1 from catfish testis we performed $5^{\prime}$ and $3^{\prime}$ RACE using the partial dmrt11 cDNA sequence data that was previously obtained from C. gariepinus (Raghuveer et al. 2005). After aligning the $5^{\prime}$ and $3^{\prime}$ end regions of $d m r t 1$ sequence that overlap in the DM-domain region, we obtained $1 \cdot 1 \mathrm{~kb}$ full-length cDNA sequence of $d m r t 1$ (hereafter referred as dmrtla) which encodes a putative protein of 287 amino acids (Fig. 1A). The amino acid sequence comparison of catfish dmrtla with other vertebrate dmrtl proteins revealed considerable homology with other teleosts like zebrafish $(61 \%)$, rainbow trout $(59 \%)$, the eel $(57 \%)$ and the Nile tilapia (54\%). The phylogenetic analysis of

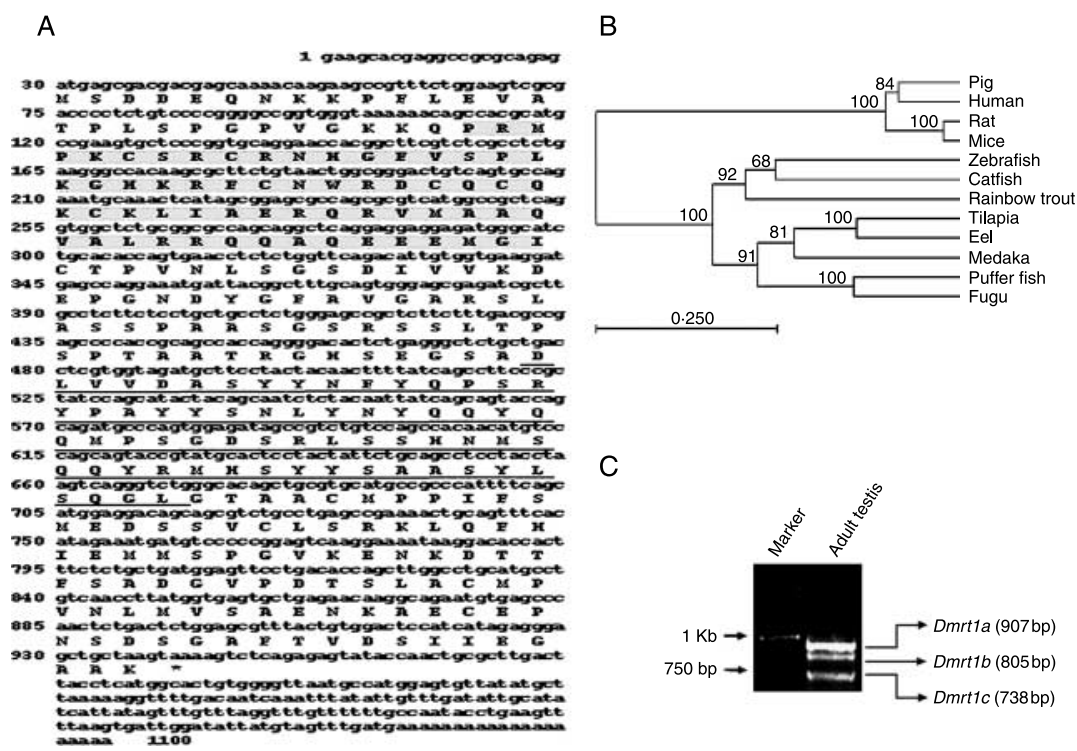

Figure 1 (A) Nucleotide sequence of catfish full-length dmrt1a cDNA and its deduced amino acid sequence. Nucleotides are numbered to the left. The DM domain is shaded, putative SY domain is underlined, *indicates stop codon. (B) Phylogenetic tree showing the evolutionary status of catfish dmrt1. Bootstrap analysis with 100 replicates was used to assess the strength of nodes in the tree. Phylogenetic analysis was done using CLC bio: CLC Main workbench software (accession numbers: Human NM_015826; Mice NM_021951.2; Rat NM_053706; Pig NM_214111; Zebrafish AY157562; Medaka AF319994; Rainbow trout NM_001124269; Eel AF421347; Fugu NM_001037949; Tilapia AF209095; Pufferfish AY135175; Catfish (dmrt1a) FJ596554). (C) Representative gel image showing multiple alternative spliced forms of catfish dmrt 1 amplified from adult testis by RT-PCR using ORF flanking primers. 
vertebrate Dmrt1 proteins (Fig. 1B) showed the existence of two main clades. The first clade represents mammalian Dmrtl sequences while the second one is for teleost Dmrtl sequences. Interestingly, we obtained multiple $d m r t 1$ transcripts (Fig. 1C) when we attempted to amplify the ORF region of dmrt1 using specific primers designed at ORF flanking region of full-length $d m r t$ la cDNA. Sequence information of these multiple bands revealed that these products represented dmrt1 transcripts generated by multiple alternative splicing. Two alternative spliced forms $d m r t 1 \mathrm{~b}$ and $d m r t 1 \mathrm{c}$ were obtained in catfish along with dmrtla, which encodes different size predicted proteins with 253, 233 and 287 amino acids respectively (Fig. 2). The nucleotide sequence data of multiple $d m r t 1$ s have been submitted to the GenBank and the accession numbers are dmrtla (FJ596554), dmrtlb (FJ596555) and dmrtlc (FJ596556). The entire alternative splicing events of the dmrt1 in catfish occurred within the ORF towards the $5^{\prime}$ end region. Interestingly, the $d m r t 1 c$ isoform is lacking most of the DM-domain region at $5^{\prime}$ end. Cloning of $d m r t 1 \mathrm{a}$ and $d m r t 1 \mathrm{c}$ ORFs from a closely related species, C. batrachus confirmed our findings in C. gariepinus. The GenBank accession numbers of $C$. batrachus dmrtls are FJ596557 and FJ596558.

\section{Tissue distribution and expression pattern of dmrt1 in adult and developing gonads}

Tissue distribution pattern of dmrt1 revealed exclusive expression in testis (Fig. 3A). Semi-quantitative RT-PCR analysis showed that $d m r t 1$ spliced forms a, b and c were detectable only in adult testis (Fig. 3B). Further the expression of $d m r t l \mathrm{a}$ was higher than those of $d m r t \mathrm{lb}$ and dmrt1c. Stage-dependent increase in $d m r t 1 \mathrm{a}$ expression was observed in testis at different age groups of catfish (50, 100 and 150 dah). Expression of dmrt1b and $d m r t 1 \mathrm{c}$ were also evident in developing male gonads. By contrast, dmrtla could not be detected in developing female gonads (Fig. 3C).

\section{Dmrt1 expression in different phases of testicular cycle}

Real-time qRT-PCR analysis showed that dmrtla and $d m r t$ lb expression was high during pre-spawning when compared with preparatory, spawning and post-spawning phases. A similar kind of expression pattern was true for dmrtlc which lacks the majority of the DM-domain (Fig. 4). This indicates that $d m r t 1$ transcripts are abundantly expressed during active spermatogenesis i.e. in preparatory and pre-spawning phases where spermatogonia and spermatocytes are more in number (Swapna et al. 2006). In addition, dmrtla transcript is abundantly expressed in testis when compared with $d m r t 1 \mathrm{~b}$ and dmrtlc transcripts. Studies from semiquantitative RT-PCR pilot experiments revealed similar patterns of expression for multiple dmrtls (densitometric quantification data not shown). Representative gel image was shown in the inlet of Fig. 4.

\section{Immunocytochemical and immunoflouresence detection of $d m r t 1$ in testis}

The polyclonal catfish $d m r t 1$ antibody raised in rabbit revealed an expected band of $\sim 31 \mathrm{kDa}$ by western blotting in testis but not in other tissues. This band

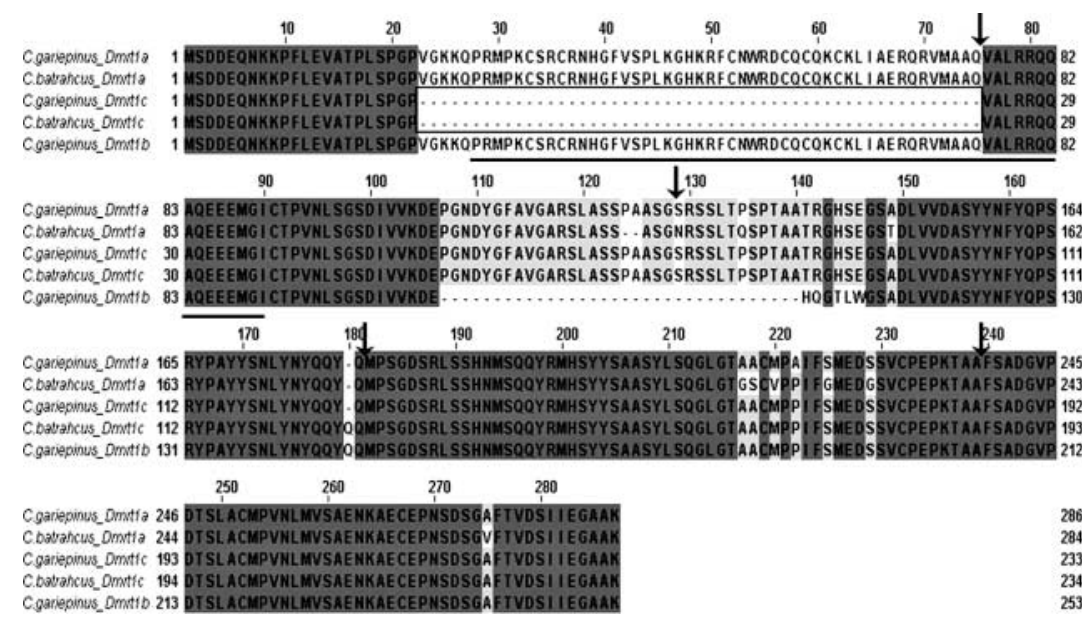

Figure 2 Amino acid sequence alignment of multiple dmrt1s from Clarias gariepinus and $C$. batrachus using EBI clustalW software. GenBank accession numbers ( $C$. gariepinus: dmrt1a (FJ596554), dmrt1b (FJ596555), dmrt1c (FJ596556) and C. batrachus: dmrt1a (FJ596557), dmrt1c (FJ596558)). DM-domain is underlined. Box indicates loss of DM-domain due to alternative splicing in $d m r t 1 c$. Arrows showing splicing sites of the dmrt1 intron-exon junctions. 
A

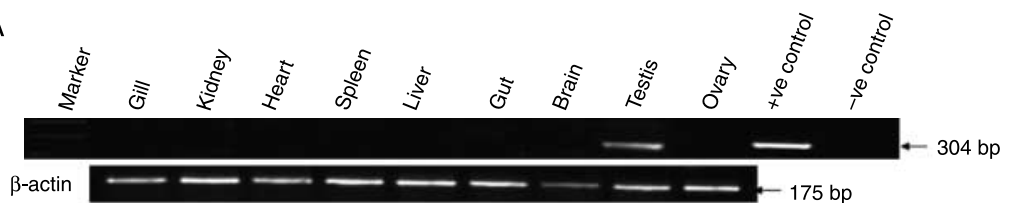

B

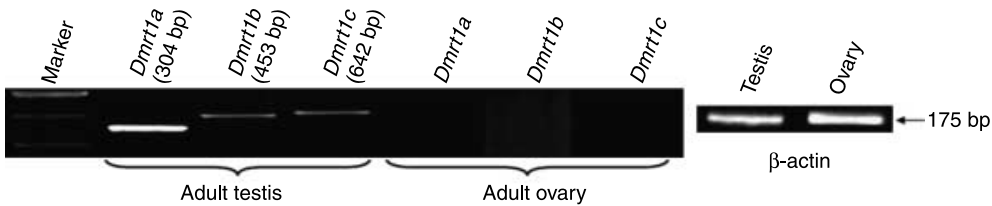

C
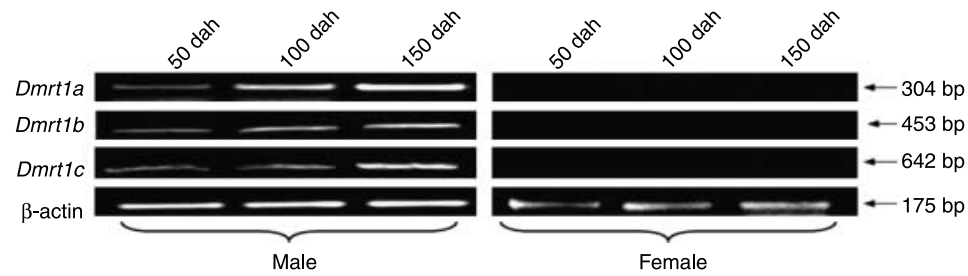

Figure 3 (A) RT-PCR analysis in different tissues of adult catfish. (B) Semiquantitative RT-PCR analysis $(n=3)$ of multiple dmrt1s in adult testis and ovary.

(C) Semi-quantitative RT-PCR $(n=3)$ amplification of multiple dmrt1s in testis and ovary at different age groups of juvenile catfish (50,100 and 150 days after hatching). $\beta$-actin was used as an internal endogenous control.

corresponds to the dmrtla form which encodes a $31 \mathrm{kDa}$ putative protein (Fig. 5). However, we could not detect the other two variants of $d m r t 1$ by western blot which may be due to their low expression levels as mentioned in the real-time PCR results. Absence of immunoreactivity using pre-absorbed antiserum confirms that the primary antibody of dmrt1 is specific for catfish (Fig. 6A). Our immunocytochemical data revealed that $d m r t 1$ expression was observed in the surrounding regions of the testicular lumens filled with spermatozoa (Fig. 6B). At higher magnification, dmrt1 immunoreactivity was detected in primary spermatogonia (SG1), secondary spermatogonia (SG2) and spermatocytes (SC), while spermatids/sperm (SP) did not show any immunoreactivity in both mature testis (Fig. 6C and D) and juvenile testis at 200 dah (Fig. 6E). We also carried out immunofluoresence studies, where $d m r t 1$ specific immunofluorescence signal (green) was detected in spermatogenic cells surrounding the lumens of adult testis except SP (Fig. $6 \mathrm{~F}$ and $\mathrm{G}$ ). But no dmrt 1 signal was detected in the pre-vitellogenic oocytes (PO) of ovary (Fig. 6H). Red fluorescence is due to PI indicating SP.

\section{Histological observation of gonadal development in catfish and gonads of MT-treated fishes}

Conventional histological methods were used to study the onset of gonadal sex differentiation in catfish.

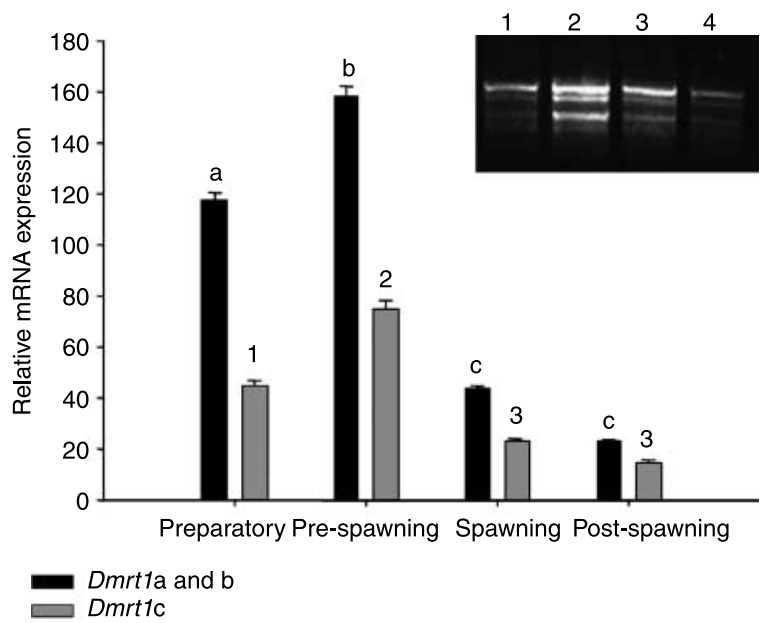

Figure 4 Real-time quantitative RT-PCR analysis of multiple dmrt1s during different phases of testicular cycle in catfish.

The relative expression of multiple dmrt1s to $\beta$-actin in gonads was analyzed by the Applied Biosystems software. Data from real-time PCR were expressed as mean \pm S.E.M. $(n=3)$. Common letters/numbers indicate means that are not significantly different. Means with different letters/numbers differ significantly $(P<0.05)$. Significance between groups was tested by ANOVA followed by Student's-Newman-Keuls' test using Sigma stat 3.5 software. Inlet of Fig. 4: Representative gel image showing semi-quantitative RT-PCR expression of multiple dmrt1s during different phases of testicular cycle (i. preparatory, ii. pre-spawning, iii. spawning and iv. post-spawning phases; $n=3$ ). 


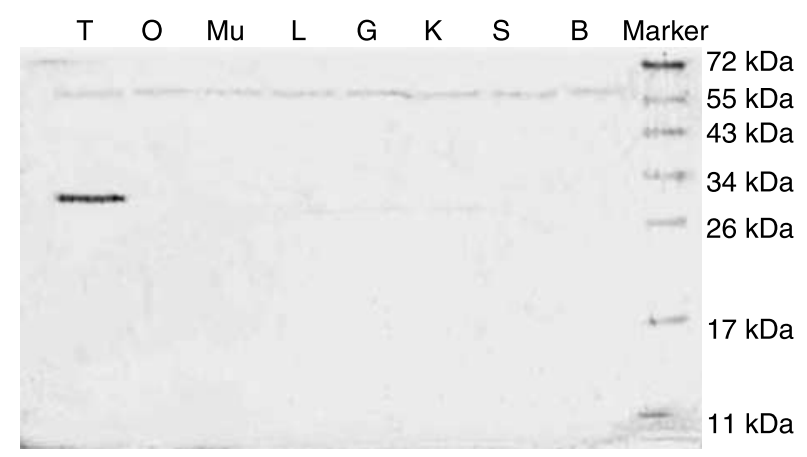

Figure 5 Western blot analysis (top panel) of Dmrt1 protein expression in various tissues, such as heart $(\mathrm{H})$, muscle $(\mathrm{Mu})$, liver $(\mathrm{L})$, kidney $(\mathrm{K})$, spleen $(\mathrm{S})$, brain $(\mathrm{B})$, ovary $(\mathrm{O})$ and testis $(\mathrm{T})$ which revealed an $\sim 31 \mathrm{kDa}$ Dmrt1 protein band in testis, which is consistent with the size calculated from the sequence (31 $224 \mathrm{kDa}$ ) using Lasergene software.

A primitive gonad with primordial germ cells surrounded by supporting cells was observed in the abdomen region near coelomic cavity at 30 dah (Fig. 7A). Histological observations of gonadal development in catfish revealed that ovarian differentiation precedes testicular differentiation. Female gonad at 50 dah (Fig. 7B) showed signs of development of ovarian cavity (OC) and few growing oocytes (Goc) already. The ovarian differentiation is completed at 75 dah showing PO (Fig. 7C) while testicular differentiation in catfish started around 50-75 dah wherein developing germ cells were observed (Fig. 7D and E). Differentiated gonads can be isolated from 75-day-old fingerlings and the proliferation of spermatogonia (SG) and oogonia (OG) can be observed at this age. The testicular development was clearly evident in 100 dah gonadal sections showing developing SG1, and SG2 (Fig. 7F). In male gonad at 200 dah, the lumens are filled with few SP indicating the progress of spermatogenesis (Fig. 7G). Female gonad at 150 dah showed PO (Fig. $7 \mathrm{H})$. These observations indicated that morphological gonadal sex differentiation occurs around $40-50$ dah as determined by the formation of an OC and the proliferation of OG. These events began earlier than the proliferation of SG.

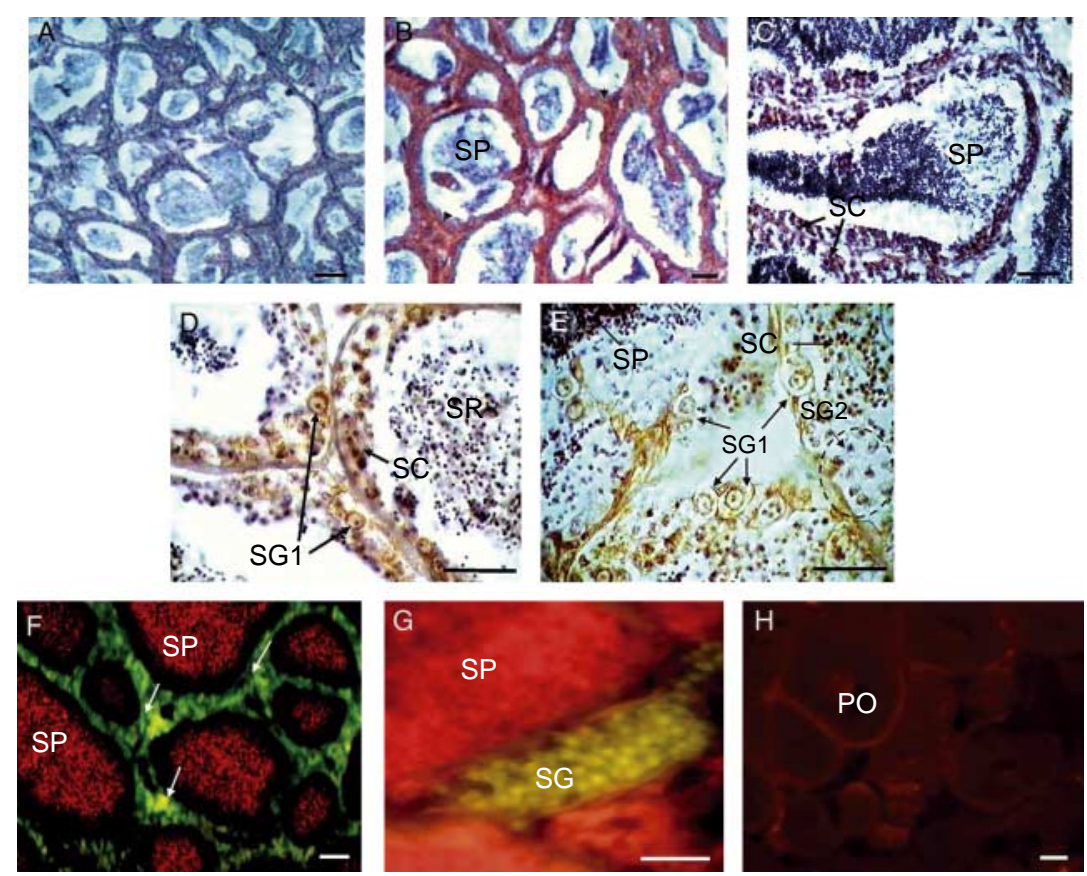

Figure 6 Immunocytochemical localization of $d m r t 1$ in adult and juvenile testis using anticatfish dmrt1 serum. (A) Adult testis section incubated with pre-absorbed antiserum, which was used as a negative control. (B) Adult testis section showing dmrt1 immunoreactivity in boundaries of lumens filled with spermatids/sperm (SP); arrow heads show DAB staining. (C) and (D) Adult testis section at higher magnifications. (E) Juvenile catfish testis section at 200 dah. Arrows indicate the position of the cell types: primary spermatogonia (SG1), secondary spermatogonia (SG2), spermatocytes (SC), sperma$\mathrm{tid} / \mathrm{sperm}$ (SP), counter stained with hematoxylin. Immunofluoresence localization of $d m r t 1$ in adult testis using anti-catfish dmrt1 serum. $(F)$ and $(G)$ Adult testis section: arrows indicate the position of the spermatogenic cells. $(\mathrm{H})$ Ovary section: previtellogenic oocyte $(\mathrm{PO})$, counter stained with propidium iodide. All scale bars represent $50 \mu \mathrm{m}$. Full colour version of this figure available via http://dx.doi.org/10.1677/JME-09-0011. 

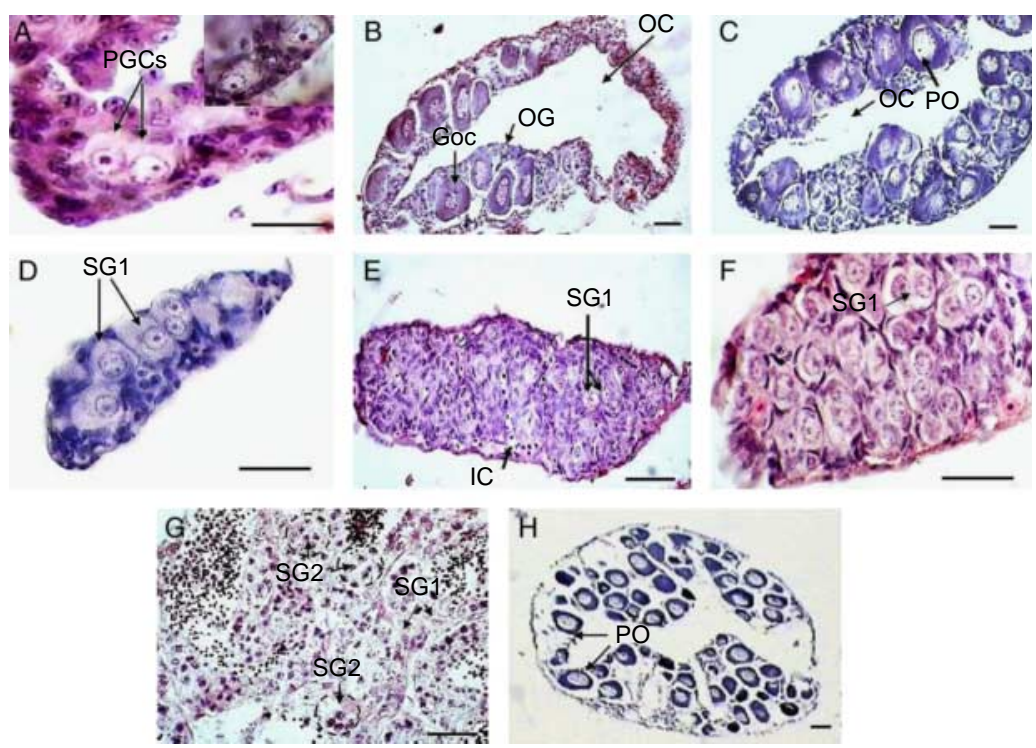

Figure 7 The development and differentiation of catfish gonads (hematoxylin-eosin staining). (A) 30 dah primitive gonad showing primordial germ cells (PGCs). Inlet showing another area where two PGCs are surrounded by supporting cells. (B) and (C) 50 dah and 75 dah female gonads. (D) and (E) 50 dah and 75 dah male gonads. $(F)$ and $(G) 100$ dah and 200 dah male gonads. (H) 150 dah female gonad. Arrows indicate the position of the cells: primordial germ cells (PGCs), primary spermatogonia (SG1), secondary spermatogonia (SG2), spermatocytes (SC), spermatid/sperm (SP), interstitial cells (IC), oogonia (OG), growing oocyte (Goc), pre-vitellogenic occyte (PO) and ovarian cavity (OC); broken circles indicate group of SG2 in Fig. 7G. All scale bars represent $50 \mu \mathrm{m}$. Full colour version of this figure available via http://dx.doi.org/10. 1677/JME-09-0011.

Histological examination of gonads of MT-treated fishes $(80 \%)$ revealed development of mature males with testes. However, $20 \%$ of the fish population appeared as intersex/bisexual, which is evident by the presence of ova-testis and ovary (Fig. 8A). In the same group, few fish had testis and ovary. On further evaluation, the cross-section of ova-testis showed the presence of spermatogenic cells, sperm and oocytes (Fig. 8B). Histological section of the mature ovary showed full grown vitellogenic follicles as well as previtellogenic follicles (Fig. 8C). Representative histological examination of testis section from $80 \%$ of the group of mature males revealed the presence of various types of spermatogenic cells (Fig. 8D).

\section{Expression of dmrt1, sox9a, foxl2 and cyp19a1 in gonads of MT-treated fishes}

Ova-testis gonad of MT-treated fish showed expression of all the genes tested, i.e. $d m r t 1$, sox $9 a$, foxl2, cyp19a1 (Fig. 9) as it contained both ovarian follicles and spermatogenic cells. On the other hand, MT-treated fishes with mature testes showed the expression of $d m r t 1$ and sox9a only (Fig. 9). Foxl2 and cyp19a1 expression are restricted to mature ovary of
MT-treated fish, which possess pre-vitellogenic and mature vitellogenic follicles (Fig. 9). The quality of first strand cDNA templates obtained from MT-treated gonads was tested using $\beta$-actin (data not shown).
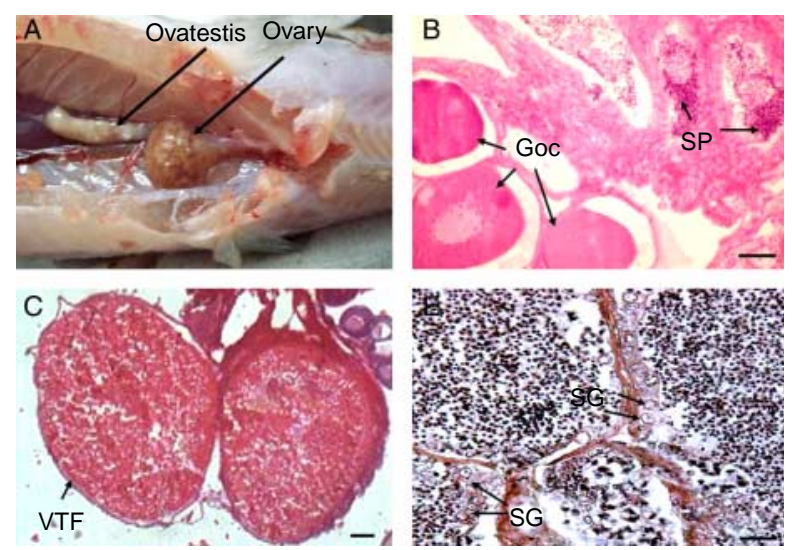

Figure 8 (A) Representative photograph of MT-treated fish showing morphology of ova-testis and ovary. Histology of MT-treated fish gonads (hematoxylin-eosin staining): (B) Ovatestis, (C) Mature ovary, (D) Mature testis. Arrows indicate the position of the cells: spermatogonia (SG), spermatocytes (SC), spermatid/sperm (SP) growing oocyte (Goc) and Vitellogenic follicle (VTF). All scale bars represent $50 \mu \mathrm{m}$. Full colour version of this figure available via http://dx.doi.org/10.1677/JME-09-0011. 


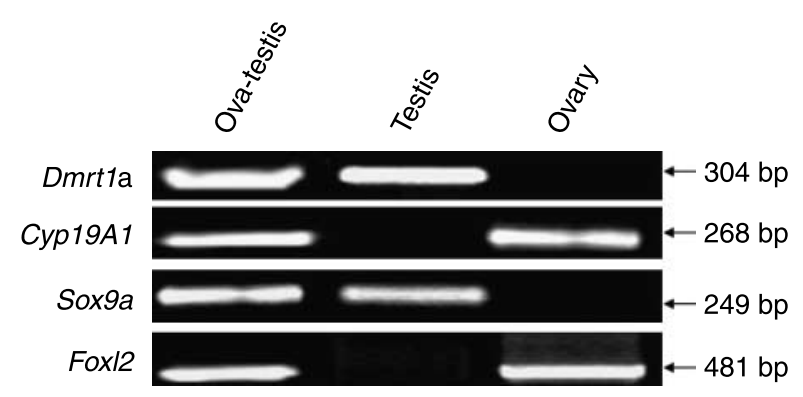

Figure 9 Representative gel image showing expressions of dmrt1a, sox9a, foxl2 and cyp19a1 in ova-testis, mature ovary and mature testis of MT-treated fishes.

\section{Discussion}

This report depicts cloning of multiple forms of $d m r t 1$ in C. gariepinus. We observed the expression pattern of $d m r t 1$ in developing testis and ovary by semi-quantitative RT-PCR and also during different phases of testicular cycle by real-time qRT-PCR. Among multiple $d m r t 1 \mathrm{~s}$, dmrtla, the dominant form, was obtained using RACE. It showed a high degree of homology with other vertebrate species at the amino acid level in the DM domain region which is conserved across different phyla (Raymond et al. 1998). In addition to dmrtla, we report the isolation of two different alternative spliced forms, $d m r t 1 \mathrm{~b}$ and $d m r t 1 \mathrm{c}$ from adult catfish testis using RT-PCR amplification with ORF flanking primers. All splicing events occurred within the ORF region at the $5^{\prime}$ end. Interestingly, $d m r t 1 c$ lacks a major part of the DM-domain region. Multiple alternative splicing in dmrt1 gene was familiar in diverse species (Burtis \& Baker 1989, Sreenivasulu et al. 2002, Guo et al. 2005, Cheng et al. 2006, Lu et al. 2007, Zhao et al. 2007). To our knowledge, $5^{\prime}$ end spliced variants were shown for the first time in a lower vertebrate species in this study. Similar kinds of splicing events were also reported in the gonads of mouse, where four different forms of Dmrt1 exist and one of the forms is lacking the entire DM-domain region (Lu et al. 2007). This shows that the multiple alternative splicing, which give rise to different isoforms, is a common feature of the Dmrt1 gene in vertebrates. The functional significance of alternative splicing events is unclear at present. Alternative spliced isoforms may provide various targets for different upstream and downstream interacting factors in sexual differentiation. Few targets that are regulated by DM-factors have been identified, for example, yolk protein genes in Drosophila and Caenorhabditis elgans, which is regulated by DSX and MAB-3 respectively (Yi \& Zarkower 1999, Yi et al. 2000). The alternative spliced forms of $d m r t 1 \mathrm{~b}$ or $d m r t 1 \mathrm{c}$ may regulate the activity of $d m r t$ la which is dominant. Although the isoform $d m r t 1 \mathrm{c}$ is lacking most of the DM domain region, the presence of other regions (SY domain) may prevent the DNA binding of dominant isoform especially dmrtla, thus acting as a negative regulator. Further identification of new targets associated with dmrt1 will provide more information on how these spliced forms operate and regulate each other.

Tissue distribution pattern indicated exclusive expression of dmrtla in testis. This was also true when analyzed for Dmrtl protein by western blot. Semi-quantitative RT-PCR analysis of multiple $d m r t 1 \mathrm{~s}$ further confirmed sexual dimorphism. Histological studies from our laboratory revealed that the gonadal sex differentiation in catfish starts around 40-50 dah. Based on this, we analyzed multiple $d m r t 1$ s expression pattern in developing gonads between 50 and 150 dah. Multiple forms of dmrt1 exist in developing testis but not in developing ovary of catfish. Male-specific expression of Dmrt1 was noticed in some species like human (Moniot et al. 2000), chicken (Nanda et al. 1999), frog (Osawa et al. 2005), garden lizard (Sreenivasulu et al. 2002) and some fish species (Fernandino et al. 2006, Ijiri et al. 2008, Xia et al. 2007, Kobayashi et al. 2008). In our study, we observed male-specific expression of multiple $d m r t 1$ s in developing and adult catfish. Taken together, these results indicate an important role for dmrtls in an early testicular development and recrudescence (see below). By contrast, reports from zebrafish (Guo et al. 2005) and rice field eel (Huang et al. 2005) showed the expression of $d m r t 1$ in both male and female gonads. Nevertheless, the role of $d m r t 1$ in ovarian differentiation has not been defined properly in vertebrates.

The fate of $d m r t 1$ after testicular differentiation i.e. in adult testis has not been studied in detail so far in any of the lower vertebrates. In the present study, we quantified multiple $d m r t 1 \mathrm{~s}$ at different phases of testicular cycle of catfish. Our real-time PCR data showed that dmrtla and b was expressed abundantly during preparatory and pre-spawning phases where spermatogonia and spermatocytes are dominant (Swapna et al. 2006), when compared with other phases of catfish testicular cycle. This indicates that in catfish, expression of dmrt1 is higher during the period of spermatogenesis and its expression decreases gradually thereafter during spawning/spermiation and postspawning phases. In rainbow trout (Marchand et al. 2000) and pejerrey (Fernandino et al. 2006), dmrt1 expression was found to be high throughout spermatogenesis but decreased at spermiation. Seasonal changes in dmrt1 expression may contribute to the maintenance of testicular cycle. Furthermore, immunocytochemical and immunofluorescence data revealed that $d m r t 1$ was localized in primary spermatogonia, secondary spermatogonia and spermatocytes, while spermatid/spermatozoa did not show any immunoreactivity. Similar expression profile of $d m r t 1$ was 
observed in zebrafish (Guo et al. 2005), red-spotted grouper (Xia et al. 2007). Localization pattern and up-regulation of dmrt1 expression during testicular recrudescence indicates plausible role in spermatogenesis but not in spermiation/spawning.

This study also aimed to find out whether treatment of MT induces testis-specific expression pattern of $d m r t 1$ as observed in genetic female sex population of the Nile tilapia (Kobayashi et al. 2008). Exogenous hormone treatments during the critical period of sex differentiation resulted in complete sex change in the Nile tilapia (Nagahama 2005). Treatment of MT (1000 ng/l) in zebrafish and long term exposure to EE2 (5 ng/l) in eel during gonadal development resulted in intersex fish (Orn et al. 2003, Nash et al. 2004). A previous report (Raghuveer et al. 2005) from our laboratory showed that exogenous MT treatments given to catfish fries skewed the catfish population completely towards male development during the course of gonadal differentiation. However, in the present study, we obtained both masculanized and intersex/bisexual fish. The apparent discrepancy may be due to large sample size and also follow-up until adult. The dosage of MT used in the present study might also contribute to this effect. Expression of both male (dmrt1 and sox9a) and female (foxl2 and cyp19a1) sex-specific genes was observed in ova-testis gonad of MT-treated fish. On the contrary, dimorphic expression pattern of male and female sexspecific genes were observed in completely developed testis and ovary. Such dimorphic expression pattern was also reported during gonadal sex differentiation and/ or hormone-induced sex reversal (Alam et al. 2008, Kobayashi et al. 2008, Ijiri et al. 2008). By contrast, in rainbow trout oestrogen treatment up-regulates female specific genes but does not suppress all male specific genes during male-to-female gonadal trans-differentiation (Vizziano-Cantonnet et al. 2008). These results together warrant a role for $d m r t 1$ in testicular differentiation/spermatogenesis.

In conclusion, we identified multiple forms of $d m r t 1$ from adult catfish testis. Stage-dependent elevation of $d m r t 1$ s expression in juveniles authenticates a pivotal role for $d m r t 1$ in testicular differentiation. MT-treatment studies further endorse $d m r t 1$ as a testis-specific gene. Dmrt1 could be localized in SG and SC but not in SP. High expression of $d m r t 1$ during testicular recrudescence indicates its importance in the entraining of testicular cycle.

\section{Declaration of interest}

We declare that there is no conflict of interest that could be perceived as prejudicing the impartiality of the research reported.

\section{Funding}

A Grant-in-Aid (BT/PR4567/AAQ/03/219/2003) to B Senthilkumaran from Department of Biotechnology, India supported this work completely.

\section{Acknowledgements}

We also acknowledge DST-FIST and UGC-SAP programs. KR acknowledges Council of Scientific and Industrial Research, India for a Senior Research Fellowship. We sincerely thank Dr J Bogerd, Department of Endocrinology, Utrecht University, The Netherlands and Prof. Y Nagahama, National Institute for Basic Biology, Japan for their technical help during early stages of work. We also acknowledge Prof. Aparna Dutta-Gupta for lending her laboratory facilities at various stages of the work. Dr K C Majumdar and Dr K Thangaraj, Centre for Cellular and Molecular Biology, Hyderabad is gratefully acknowledged for their technical help. We thank our Vice-Chancellor, Prof. Seyed E Hasnain and the Dean, Prof. A S Raghavendra, for allowing us to use the genomics and microarray facility of School of Life Sciences, University of Hyderabad. We sincerely thank the reviewer for his invaluable suggestions in improving the manuscript considerably during revision.

\section{References}

Alam MA, Kobayashi Y, Horiguchi R, Hirai T \& Nakamura M 2008 Molecular cloning and quantitative expression of sexually dimorphic markers Dmrtl and Foxl2 during female-to-male sex change in Epinephelus merra. General and Comparative Endocrinology 157 75-85.

Burtis KC \& Baker BS 1989 Drosophila double sex gene controls somatic sexual differentiation by producing alternatively spliced mRNAs encoding related sex-specific polypeptides. Cell 56 997-1010.

Cheng HH, Ying M, Tian YH, Guo Y, McElreavey K \& Zhou RJ 2006 Transcriptional diversity of DMRT1 (dsx- and mab3-related transcription factor 1) in human testis. Cell Research 16 389-393.

Fernandino JI, Guilgur LG \& Somoza GM 2006 Dmrt1 expression analysis during spermatogenesis in pejerrey, Odontesthes bonariensis. Fish Physiology and Biochemistry 32 231-240.

De Grandi A, Calvari V, Bertini V, Bulfone A, Peverali G, Camerino G, Borsani G \& Guioli S 2000 The expression pattern of a mouse doublesex-related gene is consistent with a role in gonadal differentiation. Mechanism of Development 90 323-326.

Guan G, Kobayashi T \& Nagahama Y 2000 Sexually dimorphic expression of two types of DM (Doublesex/Mab-3)-domain genes in a teleost fish, the tilapia (Oreochromis niloticus). Biochemical and Biophysical Research Communications 272 662-666.

Guo Y, Cheng H, Huang X, Gao S, Yu H \& Zhou R 2005 Gene structure, multiple alternative splicing, and expression in gonads of zebrafish Dmrt1. Biochemical and Biophysical Research Communications 330 950-957.

Huang X, Guo Y, Shui Y, Gao S, Yu H, Cheng H \& Zhou R 2005 Multiple alternative splicing and differential expression of dmrtl during gonad transformation of the rice field eel. Biology of Reproduction 73 1017-1024.

Hughes IA 2001 Minireview: sex differentiation. Endocrinology 142 3281-3287.

Ijiri S, Kaneko H, Kobayshi T, Wang DS, Sakai F, Paul-Prasanth B, Nakamura M \& Nagahama Y 2008 Sexual dimorphic expression of genes during early differentiation of a teleost fish, the Nile tilapia Oreochromis niloticus. Biology of Reproduction 78 333-341.

Kettlewell JR, Raymond CS \& Zarkower D 2000 Temperaturedependent expression of turtle Dmrt1 before sexual differentiation. Genesis 26 174-178. 
Kobayashi T, Matsuda M, Kajiura-Kobayashi H, Suzuki A, Saito N, Nakamoto M, Shibata N \& Nagahama Y 2004 Two DM domain genes, DMY and DMRT1, involved in testicular differentiation and development in the medaka, Oryzias latipes. Developmental Dynamics $231518-526$.

Kobayashi T, Kajiura-Kobayashi H, Guan G \& Nagahama Y 2008 Sexual dimorphic expression of DMRT1 and Sox9a during gonadal differentiation and hormone-induced sex reversal in the teleost fish Nile tilapia (Oreochromis niloticus). Developmental Dynamics 237 297-306.

Kwon JY, McAndrew BJ \& Penman DJ 2001 Cloning of brain aromatase gene and expression of brain and ovarian aromatase genes during sexual differentiation in genetic male and female Nile tilapia Oreochromis niloticus. Molecular Reproduction and Development $\mathbf{5 9}$ 359-370.

Lu H, Huang X, Zhang L, Guo Y, Cheng H \& Zhou R 2007 Multiple alternative splicing of mouse Dmrtl during gonadal differentiation. Biochemical and Biophysical Research Communications 352 630-634.

Marchand O, Govoroun M, D'Cotta H, McMeel O, Lareyre J, Bernot A, Laudet V \& Guiguen Y 2000 DMRT1 expression during gonadal differentiation and spermatogenesis in the rainbow trout, Oncorhynchus mykiss. Biochemica et Biophysica Acta 1493 180-187.

Matsuda M, Nagahama Y, Shinomiya A, Sato T, Matsuda C, Kobayashi T, Morrey CE, Shibata N, Asakawa S, Shimizu N et al. 2002 DMY is a Y-specific DM-domain gene required for male development in the medaka fish. Nature 417 559-563.

Moniot B, Berta P, Scherer G, Sudbeck P \& Poulat F 2000 Male specific expression suggests role of DMRT1 in human sex determination. Mechanism of Development 91 323-325.

Nagahama Y 2005 Molecular mechanisms of sex determination and gonadal sex differentiation in fish. Fish Physiology and Biochemistry 31 105-109.

Nanda I, Shan Z, Schartl M, Burt DW, Koehler M, Nothwang H, Grutzner F, Paton IR, Windsor D, Dunn I et al. 1999 Three hundred million years of conserved synteny between chicken $\mathrm{Z}$ and human chromosome 9. Nature Genetics 21 258-259.

Nash JP, Kime DE, Van der Ven LT, Wester PW, Brion F, Maack G, Stahlschmidt-Allner P \& Tyler CR 2004 Long-term exposure to environmental concentrations of the pharmaceutical ethynylestradiol causes reproductive failure in fish. Environmental Health Perspectives 112 1725-1733.

Orn S, Holbech H, Madsen TH, Norrgren L \& Petersen GI 2003 Gonad development and vitellogenin production in zebrafish (Danio rerio) exposed to ethinylestradiol and methyltestosterone. Aquatic Toxicology 65 397-411.

Osawa N, Oshima Y \& Nakamura M 2005 Molecular cloning of Dmrtl and its expression in the gonad of Xenopus. Zoological Science 22 681-687.

Ottolenghi C \& McElreavey K 2000 Deletion of 9p and quest for a conserved mechanism of sex determination. Molecular Genetics and Metabolism 71 397-404.

Raghuveer K, Rahul G, Wang DS, Bogerd J, Kirubagaran R, Rasheeda MK, Sreenivasulu G, Bhattacharya N, Tarangini S, Nagahama Y et al. 2005 Effect of methyl testosterone and ethynyl estradiol-induced sex differentiation on catfish, Clarias gariepinus: Expression profiles of Dmrt1, cytochrome P450aromatases and 3 3 -hydroxysteroid dehydrogenase. Fish Physiology and Biochemistry 31 143-147.

Rasheeda MK, Sreenivasulu G, Swapna I, Raghuveer K, Wang DS, Thangaraj K, Dutta-Gupta A \& Senthilkumaran B 2005
Thiourea-induced alteration in the expression patterns of some steroidogenic enzymes in the air-breathing catfish Clarias gariepinus. Fish Physiology and Biochemistry 31 275-279.

Raymond CS, Shamu CE, Shen MM, Seifert KJ, Hirsch B, Hodgkin J \& Zarkower D 1998 Evidence for evolutionary conservation of sexdetermining genes. Nature 391 691-695.

Raymond CS, Parker ED, Kettlewell JR, Brown LG, Page DC, Kusz K, Jaruzelska J, Reinberg Y, Flejter WL, Bardwell VJ et al. 1999 A region of human chromosome $9 \mathrm{p}$ required for testis development contains two genes related to known sexual regulators. Human Molecular Genetics 8 989-996.

Raymond CS, Murphy MW, O’Sullivan MG, Bardwell VJ \& Zarkower D 2000 Dmrt 1 , a gene related to warm and fly sexual regulators, is required for mammalian testis differentiation. Genes and Development 14 2587-2595.

Sreenivasulu K, Ganesh S \& Raman R 2002 Evolutionarily conserved, DMRT1, encodes alternatively spliced transcripts and shows dimorphic expression during gonadal differentiation in the lizard, Calotes versicolor. Gene Expression Patterns 2 51-60.

Swain A \& Lovell-Badge R 1999 Mammalian sex determination: a molecular drama. Genes and Development 13 755-767.

Swapna I, Rajasekhar M, Supriya A, Raghuveer K, Sreenivasulu G, Rasheeda MK, Majumdar KC, Kagawa H, Tanaka H, Dutta-Gupta A et al. 2006 Thiourea-induced thyroid hormone depletion impairs testicular recrudescence in the air-breathing catfish, Clarias gariepinus. Comparative Biochemistry and Physiology 144 1-10.

Vizziano-Cantonnet D, Baron D, Mahe S, Cauty C, Fostier A \& Guiguen Y 2008 Estrogen treatment up-regulates female genes but does not suppress all early testicular markers during rainbow trout male-to-female gonadal trans-differentiation. Journal of Molecular Endocrinology 41 277-288.

Wang DS, Kobayashi T, Senthilkumaran B, Sakai F, Sudhakumari CC, Suzuki T, Yoshikuni M, Matsuda M, Morohashi K \& Nagahama Y 2002 Molecular cloning of DAX-1 and SHP cDNAs and their expression patterns in the Nile tilapia, Oreochromis niloticus. Biochemical and Biophysical Research Communications 297 632-640.

Xia W, Zhou L, Yao B, Li CJ \& Gui JF 2007 Differential and spermatogenic cell-specific expression of DMRT1 during sex reversal in protogynous hermaphroditic groupers. Molecular and Cellular Endocrinology 263 156-172.

Yi W \& Zarkower D 1999 Similarity of DNA binding and transcriptional regulation by Caenorhabditis elegans MAB-3 and Drosophila melanogaster DSX suggests conservation of sex determining mechanisms. Development 126 873-881.

Yi W, Ross JM \& Zarkower D 2000 Mab-3 is a direct tra-1 target gene regulating diverse aspects of C. elegans male sexual development and behavior. Development 127 4469-4480.

Zhang J 2004 Evolution of DMY, a newly emergent male sexdetermination gene of medaka fish. Genetics 166 1887-1895.

Zhao Y, Lu H, Yu H, Cheng H \& Zhou R 2007 Multiple alternative splicing in gonads of chicken DMRT1. Development Genes and Evolution 217 119-126.

Received in final form 10 February 2009

Accepted 24 February 2009

Made available online as an Accepted Preprint 24 February 2009 